www.jmscr.igmpublication.org

Impact Factor 5.84

Index Copernicus Value: 83.27

ISSN (e)-2347-176x ISSN (p) 2455-0450

crossref DOI: _https://dx.doi.org/10.18535/jmscr/v5i5.139

Journal Of Medical Science And Clinical Research

\title{
Cardiac and Extra Cardiac Predictors and Complications of Acute Atrial Fibrillation Complicating ST Elevation Myocardial Infarction (STEMI) ST Elevation myocardial infarction Acute Atrial Fibrillation (STAAF) Study
}

\author{
Authors \\ Dr Supriya D Nambiar, Dr Mathew Iype, Prof. Sunitha Viswanathan, \\ Prof. George Koshy, Prof. Prabha Nini Gupta, Prof. V.V. Radhakrishnan \\ STAAF Investigators
}

\begin{abstract}
Study Population and Design: Acute Atrial Fibrillation $(A F)$ is an important complication of ST Segment Elevation Myocardial Infarction (STEMI) even in the Primary angioplasty era. AF is an independent predictor of both short termand long term mortality. What is the incremental risk of developing complications when AF complicates STEMI?

Materials and Methods: It was a single center, prospective cohort study, conducted from October 2014 to January 2016. The patients were divided into three groups. 1. Those with AF at admission. 2. Those developing AF within 24 hours of admission. 3. Those developing AF after 24 hours and till discharge. The patient's clinical and echocardiographic profile were documented. They were followed up for development of heart failure, arrhythmias, cardiogenic shock, Cerebrovascular Accident (CVA) and death.

Results: A total of 118 STEMI patients (59 with AF and 59 without) were included in the study. In-hospital (20.3\% vs. $6.8 \% p=0.031)$ and total mortality was significantly higher in AF Group (23.7\% vs. 10.2\%, p=0.040). 1 and 3 month mortality were not significantly different in AF and non AF groups. The in-hospital MACCE rate was higher in the AF group. The 1 and 3 month MACCE were not significantly different. Pericarditis was more common in patients who had AF.(20.3\% vs. 6.8\%,p=0.03). Acute Kidney Injury was more in AF group.(16.9 \% vs. 5.1\%, p=0.04). Age >/=70, systemic hypertension, Left Ventricular EF $<40 \%$, Left Atrial dilatation, ischemic mitral regurgitation $>/=$ grade 2 were significant predictors of $A F$ on univariate analysis. Hypertension was the only independent predictor of $A F$ in multivariate analysis.

Keywords: Atrial Fibrillation, ST Elevation Myocardial Infarction, Myocardial infarction complications.
\end{abstract}

\section{Introduction}

Acute atrial fibrillation is an important complication of ST Segment Elevation Myocardial Infarction (STEMI). Acute atrial fibrillation complicates STEMI in 6.8 to $21 \%^{1,2,3,4}$. In the Fibrinolytic era, the GUSTO I trial which included 40981 patients with STEMI eligible for thrombolysis, the incidence of atrial fibrillation was $10.4 \%^{5}$. Comparable incidence of atrial fibrillation has been found in STEMI patients undergoing Primary Percutaneous Coronary Intervention (Primary PCI). In the OACIS study, among the 2475 patients with STEMI treated with Primary Percutaneous Coronary intervention 
(PCI), atrial fibrillation occurred in $12 \%$. (Kinjo et.al.) ${ }^{6}$.

In the setting of STEMI, the occurrence of AF is of particular importance since rapid and irregular ventricular rates during the arrhythmia may cause further impairment of the coronary circulation and left ventricular function. Atrial fibrillation is associated with a high mortality which may be due in part to the development of $\mathrm{AF}$ as a surrogate marker of heart failure, elevated filling pressures and atrial volume overload. Atrial fibrillation is an independent predictor of both short term $^{8,9}$ and long term mortality ${ }^{10,11 .}$

What is the incremental risk of developing complications when AF complicates STEMI? The STAAF study addresses this issue.

\section{Materials and Methods}

\subsection{Study population and design}

It was a single center, prospective cohort study, conducted from October 2014 to January 2016. The study was conducted at Government Medical College Thiruvananthapuram, a tertiary care teaching hospital in Kerala, South India. . The Institutional Review Board and the Ethics Committee approved the study protocol.

The study group consisted of patients with ST Elevation Myocardial Infarction (STEMI) having Acute Atrial Fibrillation (AF) during hospitalization phase. Exclusion criteria included chronic atrial fibrillation, rheumatic heart disease (mitral stenosis and mitral regurgitation), hypertrophic cardiomyopathy, dilated cardiomyopathy, congenital heart disease, post cardiac surgery, pulmonary hypertension, thyrotoxicosis, chronic renal failure and recent malignancy. An age and sex matched control cohort was selected from patients with STEMI without atrial fibrillation.

The sample size calculated using Pocock formula was 54 STEMI patients with Atrial fibrillation and 54 control patients with STEMI but without Atrial fibrillation.

\subsection{Definitions}

STEMI was defined as having 1. Prolonged angina within 48 hours. 2. Diagnostic electrocardiographic changes- ST segment elevation in two contiguous leads and 3. Two fold elevation in serum creatine kinase or positive high sensitivity troponin.

Atrial fibrillation was defined as an irregular rhythm with the presence of fibrillary waves, with no visible $\mathrm{p}$-waves and irregular $\mathrm{R}-\mathrm{R}$ intervals

\subsection{Methodology}

The patients were divided into three groups. 1 . Those with AF at admission. 2. Those developing AF within 24 hours of admission. 3. Those developing AF after 24 hours and till discharge

During the Coronary care stay for a minimum of 48 hours, the patient's electrocardiogram was continuously monitored. After that daily electrocardiograms were taken. The patient's clinical and echocardiographic profile were documented. They were followed up for development of new onset heart failure, bradyarrhythmias or tachyarrhythmias, cardiogenic shock, Cerebrovascular Accident (CVA) and death.

Two-dimensional echocardiography was performed with GE vivid E 9 echocardiography machine. All classic views were studied. Mitral regurgitation was quantified by pulse doppler and colour doppler.

\subsection{Statistical analysis}

The study cohort and comparison cohort were analyzed with Chi-square test and Fischer's exact test. Multivariate analysis was done for determination of independent predictors of mortality.

\section{Results}

\subsection{Patient demographics}

A total of 118 patients were included in the study. This included 59 STEMI patients with atrial fibrillation and 59 STEMI patients without atrial fibrillation. The mean age of the patients was 59.6 years. There were 47 males and 12 females in the atrial fibrillation arm and an equal number in the 
control group. Patients were grouped into $>/=70$ years and $<70$ years of age. Our study found that $18(30.5 \%)$ patients in AF group was 70 years or above where as only $8(13.6 \%)$ of non AF patients were in this age group and the difference was statistically significant $(\mathrm{p}=0.026)$.

Figure 1: Age and AF

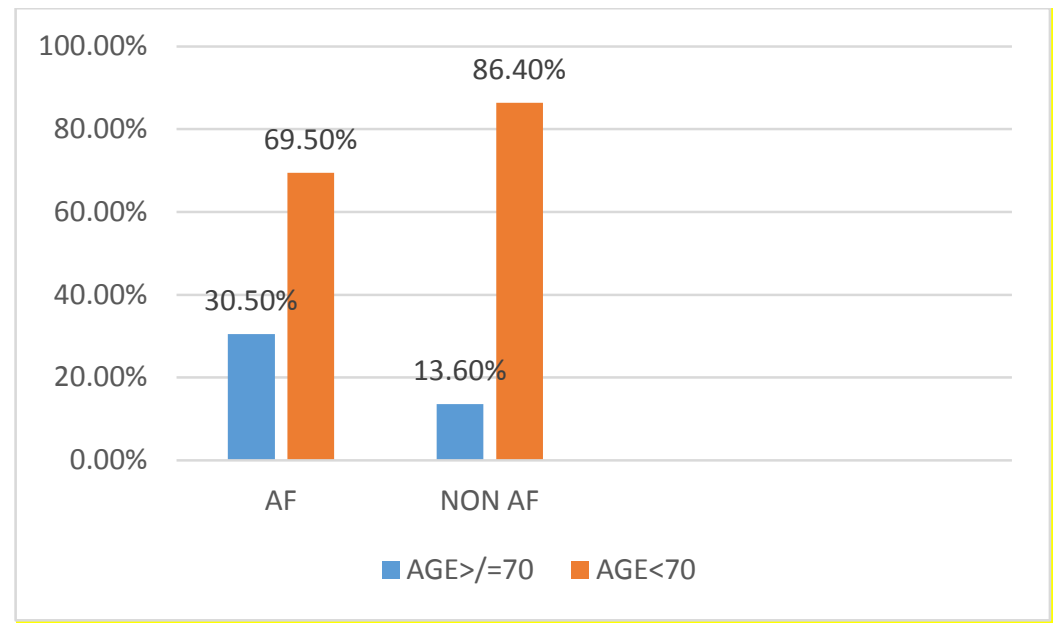

Table 1 shows the coronary artery disease (CAD) risk factors in the patient groups. There were more hypertensives in the atrial fibrillation group and this was statistically significant.

Table 1: Atherosclerotic risk factors in AF group and non- AF group

\begin{tabular}{|l|c|c|c|}
\hline & AF group & No AF group & P value \\
\hline Systemic hypertension & $38(64.4 \%)$ & $25(42.4 \%)$ & 0.016 \\
\hline Diabetes mellitus & $25(42.4 \%)$ & $28(47.5 \%)$ & 0.0579 \\
\hline Dyslipidemia & $50(84.7 \%)$ & $43(72.9 \%)$ & 0.115 \\
\hline Smoking & $35(59.3 \%)$ & $39(66.1 \%)$ & 0.446 \\
\hline
\end{tabular}

\subsection{Temporal pattern of occurrence of atrial fibrillation}

Patients in AF group was divided into AF at admission, AF within 24 hours and AF onset after 24 hours till predischarge.19 (32.2\%)patients had atrial fibrillation on admission, 30 (50.8\%) developed atrial fibrillation in 24 hours and 10 $(16.9 \%)$ patients developed atrial fibrillation after 24 hours.

AF patients were also studied in 2 groups-AF developing within 24 hours and after 24 hours of admission and prior to discharge. 49 patients had AF within 24 hours of admission (group 1) and 10 patients developed AF after 24 hours (group 2). There was no significant difference in demographic profile between 2 groups including age, sex, Diabetes mellitus, Hypertension, smoking status and Acute kidney injury.

\subsection{Nature of STEMI and reperfusion status}

More patients having inferior wall STEMI developed AF within 24 hours (59.2\%). More patients having anterior wall STEMI developed AF after 24 hours (80\%). $75 \%$ of patients who developed AF after 24 hours had $<75 \%$ ST segment resolution post - reperfusion compared to $29.8 \%$ of patients who developed AF within 24 hours. So delayed AF is more common in nonreperfused.

\subsection{Killips class on admission and AF}

More patients in AF group had higher fillip class on admission ( $22 \%$ vs. $8.5 \%, \mathrm{p}=0.041)$. See figure1. 
Figure 2: Killip's class on admission

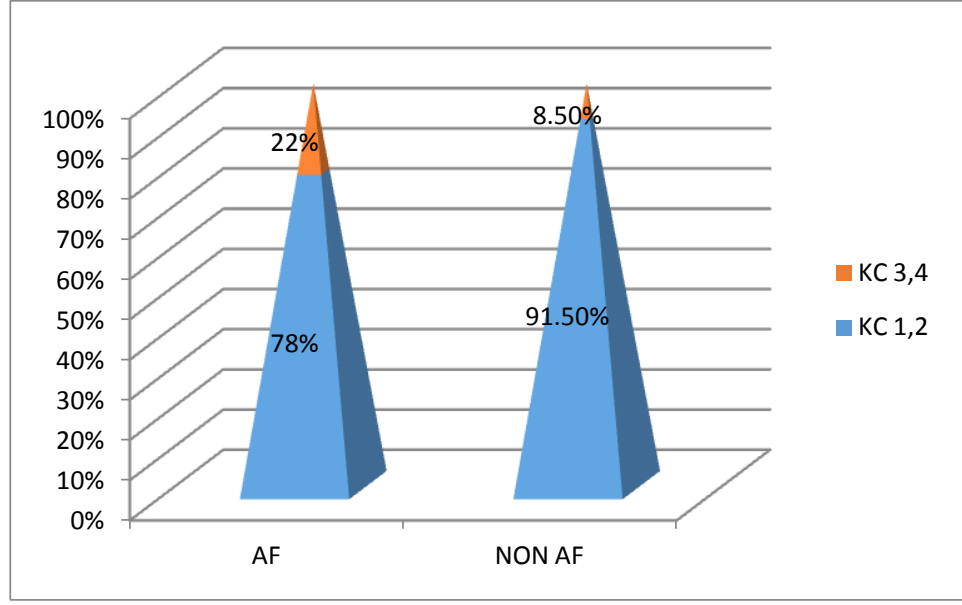

\subsection{Heart failure}

Clinical heart failure was more common in the AF group. See figure 3.

Figure 3: Heart failure

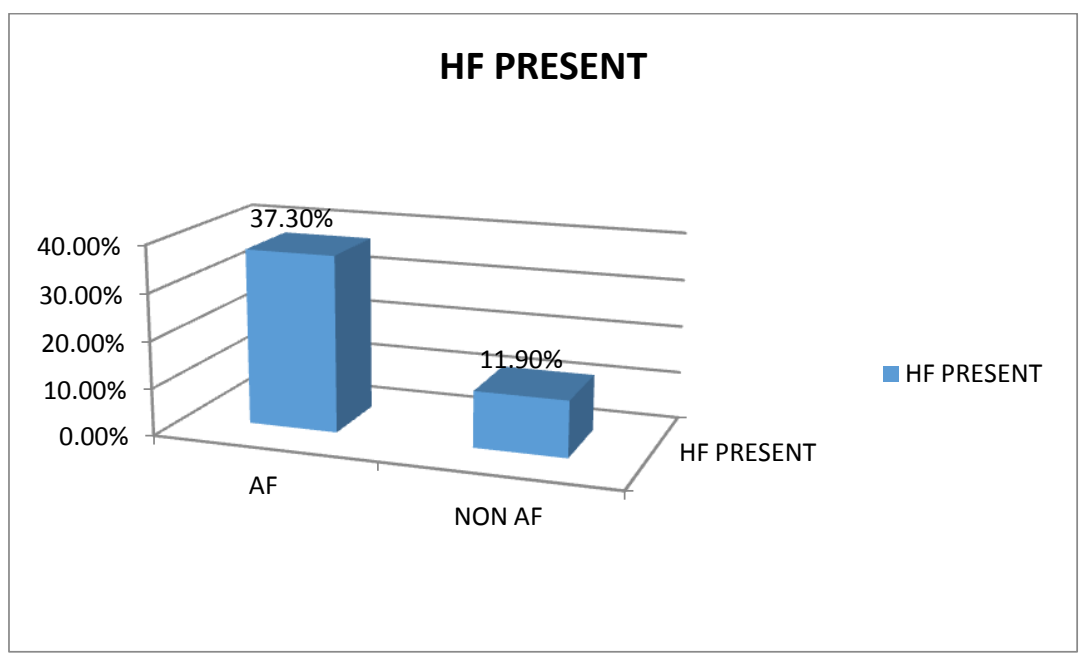

\subsection{Acute mitral regurgitation}

There was significant ischemic MR (32.2\% vs. $15.3 \% \mathrm{p}=0.030)$ in AF group. See table 2.

Table 2: Mitral regurgitation status and AF

\begin{tabular}{|l|c|c|c|}
\hline & AF group & Non AF group & P value \\
\hline MR grade $>2$ & $19(32.2 \%)$ & $9(15.3 \%)$ & 0.030 \\
\hline MR grade $</=2$ or absent & $40(67.8 \%)$ & $50(84.7 \%)$ & \\
\hline
\end{tabular}

\subsection{Pericarditis}

Pericarditis was seen in 12 patients $(20.3 \%)$ with acute AF complicating STEMI compared with 4 patients $(6.8 \%$ in those without AF. $(\mathrm{p}=0.03)$

\subsection{Acute Kidney Injury}

Acute Kidney Injury (AKI) was more in $\mathrm{AF}$ Group than non AF group(16.9\% vs. $5.1 \%$, $\mathrm{p}=0.04)$.

\subsection{Mortality}

In hospital $(20.3 \%$ vs. $6.8 \% \mathrm{p}=0.031)$ and total mortality was significantly higher in AF Group (23.7\% vs. $10.2 \%, \mathrm{p}=0.040) .1$ and 3 month mortality were not significantly different in $\mathrm{AF}$ and non AF groups. See figure 4 
Figure 4 Mortality

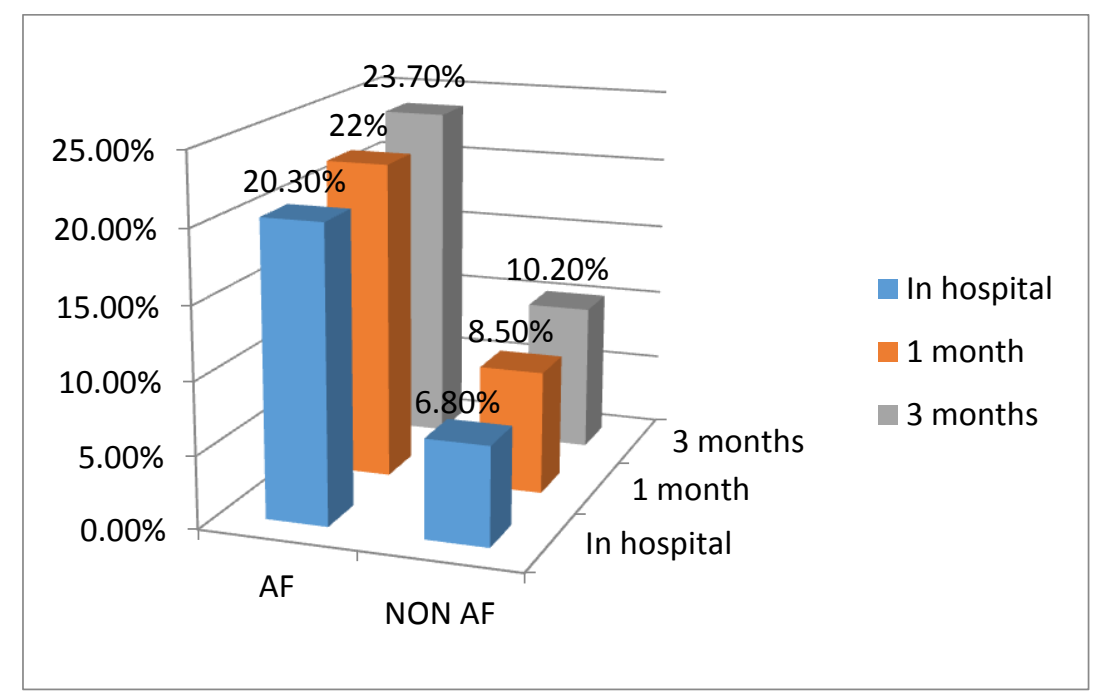

Figure 5 : Kaplan Meir survival curve

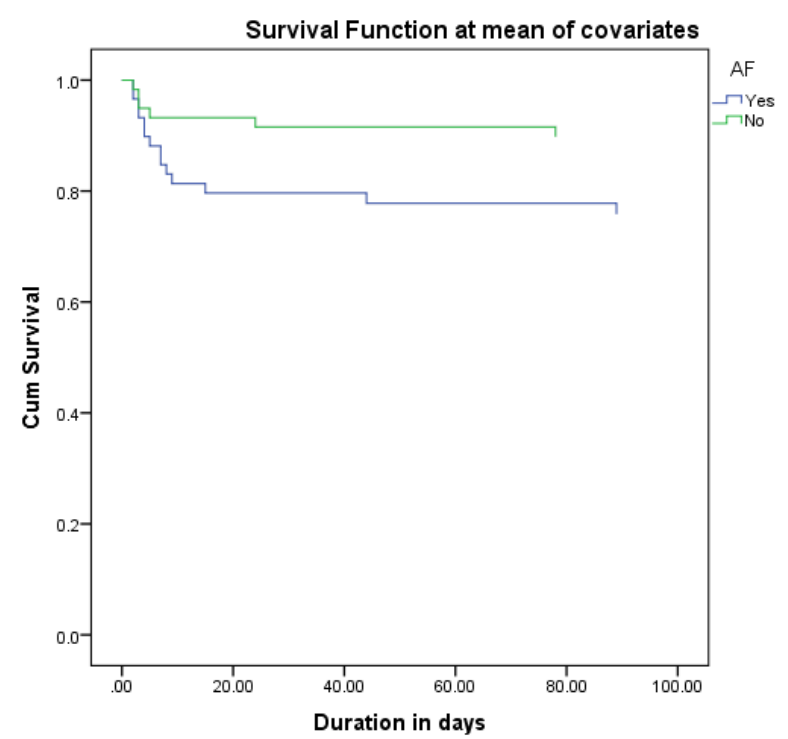

3.10 Mortality in anterior wall STEMI vs nonanterior wall STEMI

Among patients in the AF group, the in-hospital and 3 month mortality was significantly higher in patients having anterior wall STEMI compared to non- anterior wall STEMI. The 1 month mortality was also higher in the anterior wall STEMI group, but only of borderline significance.

Table 3: Mortality in anterior wall STEMI vs non- anterior wall STEMI

\begin{tabular}{|l|c|c|c|}
\hline & Anterior wall STEMI & Non-anterior wall STEMI & P value \\
\hline In-hospital mortality & $9(32.1 \%)$ & $3(9.7 \%)$ & 0.032 \\
\hline 1 month mortality & $9(32.1 \%)$ & $4(13.3 \%)$ & 0.086 \\
\hline 3 month mortality & $10(37 \%)$ & $4(13.3 \%)$ & 0.038 \\
\hline
\end{tabular}


Figure 6: Mortality in anterior wall STEMI vs. non- anterior wall STEMI

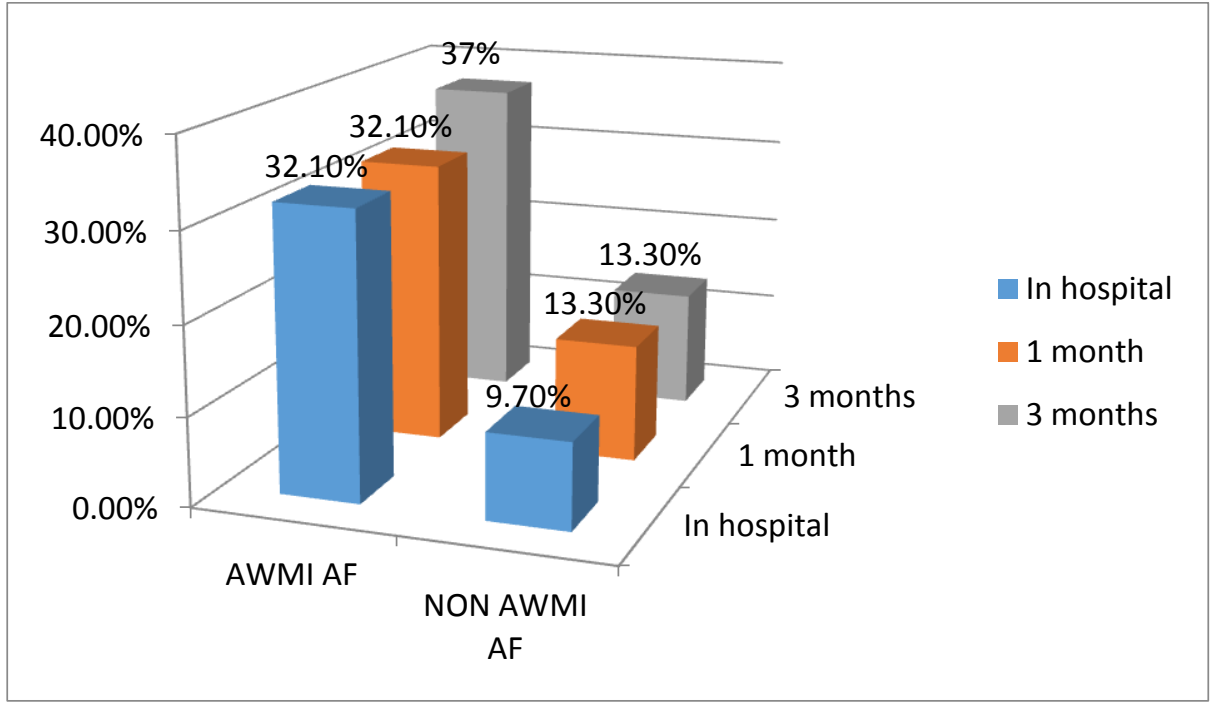

Figure 7 : Kaplan Meier survival curve of AF patients - anterior wall vs non- anterior wall STEMI

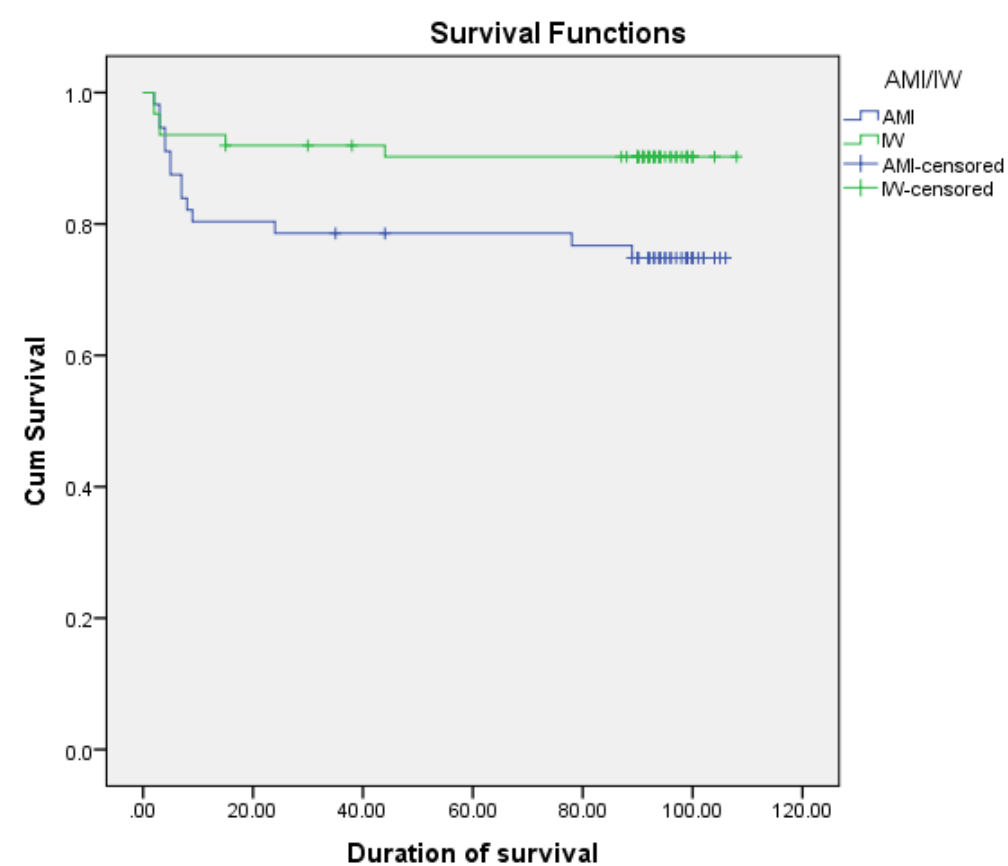

\subsection{Major Adverse Cardiac and Cerebral The 1 and 3 month MACCE were not Events (MACCE) \\ The in-hospital MACCE rate was higher in the AF significantly different in AF and non AF groups.} group compared to patients who did not have AF.

Table 5 MACCE

\begin{tabular}{|l|c|c|c|}
\hline & AF group & Non AF group & P value \\
\hline In-hospital MACCE & $29(49.2 \%)$ & $13(22 \%)$ & 0.002 \\
\hline 1 month MACCE & $12(20.3 \%)$ & $8(13.6 \%)$ & 0.048 \\
\hline 3 month MACCE & $5(8.5 \%)$ & $7(11.9 \%)$ & \\
\hline
\end{tabular}


Figure 8: MACCE

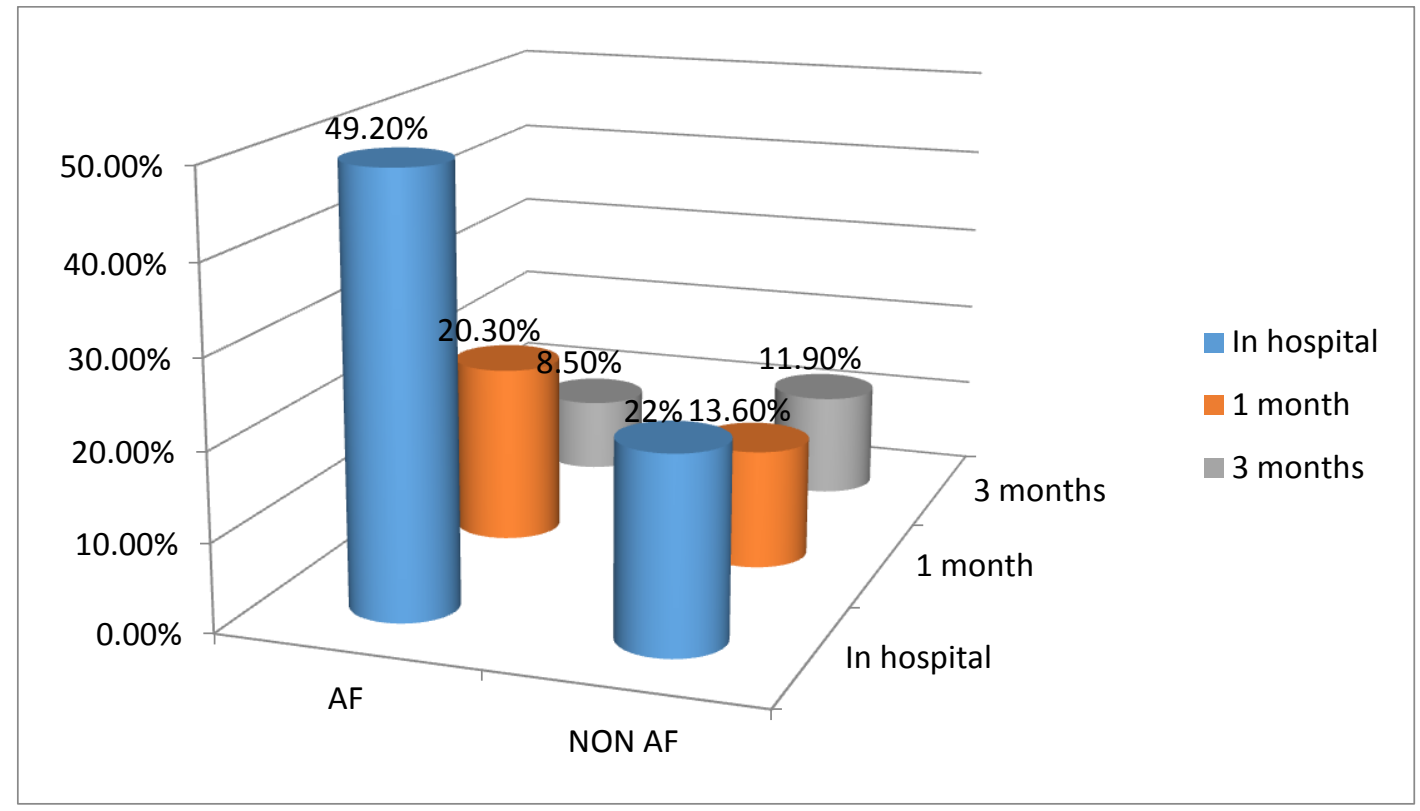

3.12 MACCE in AF group - anterior wall vs non- anterior wall

Anterior wall STEMI patients with AF had higher in-hospital, 1 month and 3 month MACCE compared to non- anterior wall STEMI.

Table 6: MACCE in AF group - anterior wall vs non- anterior wall

\begin{tabular}{|l|c|c|c|}
\hline & Anterior wall group & Non- anterior wall group & P value \\
\hline In-hospital MACCE & $18(64.3 \%)$ & $11(35.5 \%)$ & 0.027 \\
\hline 1 month MACCE & $9(47.4 \%)$ & $3(11.1 \%)$ & 0.006 \\
\hline 3 month MACCE & $5(27.8 \%)$ & 0 & 0.010 \\
\hline
\end{tabular}

\subsection{Termination of AF}

AF terminated spontaneously in $24(40.8 \%)$. It was terminated by cardio version by DC shock in
$23(38.9 \%)$ and IV amiodarone in 2 (3.4\%). 10 (16.9\%) patients remained in $\mathrm{AF}$ at the time of discharge

\begin{tabular}{|l|c|}
\hline & No (\%) \\
\hline Spontanous termination & $24(40.8 \%)$ \\
\hline DC cardioversion & $23(38.9 \%)$ \\
\hline IV Amiodarone & $2(3.4 \%)$ \\
\hline Persistant AF & $10(16.9 \%)$ \\
\hline
\end{tabular}

\subsection{Predictors of development of AF} complicating STEMI

Age $>/=70$, systemic hypertension, Left Ventricular $\mathrm{EF}<40 \%$, Left Atrial dilatation, ischemic MR $>/=$ grade2 were significant predictors of $\mathrm{AF}$ on univariate analysis. Hypertension was the only independent predictor of AF in multivariate analysis 
Table 7: Multivariate analysis of binary logistic regression on AF as dependent variable

\begin{tabular}{|l|c|c|c|}
\hline & P value & OR & 95\% CI \\
\hline Age & 0.513 & 1.453 & $0.475-4.446$ \\
\hline Systemic hypertension & 0.021 & 2.905 & $1.176-7.172$ \\
\hline Killipsclass at admission & 0.508 & 0.555 & $0.097-3.173$ \\
\hline Left atrial dilatation & 0.222 & 1.937 & $0.671-5.593$ \\
\hline Mitral regurgitation>2 & 0.703 & 0.776 & $0.211-2.855$ \\
\hline LV EF <40 & 0.9 & 0.906 & $0.194-4.232$ \\
\hline LAD involvement & 0.771 & 1.29 & $0.232-7.163$ \\
\hline Acute kidney injury & 0.258 & 2.818 & $0.469-16.941$ \\
\hline
\end{tabular}

Table 8

\begin{tabular}{|l|c|c|c|c|c|c|c|c|}
\hline \multirow{2}{*}{ Variables } & \multirow{2}{*}{$\mathrm{B}$} & S.E. & \multirow{2}{*}{ Wald } & \multirow{2}{*}{$\mathrm{df}$} & \multirow{2}{*}{$\mathrm{p}$} & \multirow{2}{*}{ OR } & \multicolumn{2}{|c|}{ 95\% C.I.for OR } \\
\cline { 6 - 10 } & & & & & & & Lower & Upper \\
\hline Age & 0.374 & 0.571 & 0.429 & 1 & 0.513 & 1.453 & 0.475 & 4.446 \\
\hline HTN & 1.066 & 0.461 & 5.346 & 1 & 0.021 & 2.905 & 1.176 & 7.172 \\
\hline TC & 0.966 & 0.466 & 4.305 & 1 & 0.038 & 2.627 & 1.055 & 6.542 \\
\hline AKI & 1.036 & 0.915 & 1.281 & 1 & 0.258 & 2.818 & 0.469 & 16.941 \\
\hline ADKC & -0.59 & 0.89 & 0.439 & 1 & 0.508 & 0.555 & 0.097 & 3.173 \\
\hline EF<40 & -0.099 & 0.787 & 0.016 & 1 & 0.9 & 0.906 & 0.194 & 4.232 \\
\hline In hospital MAC & 0.785 & 0.73 & 1.156 & 1 & 0.282 & 2.192 & 0.524 & 9.167 \\
\hline OT/ARR & 0.454 & 0.652 & 0.486 & 1 & 0.486 & 1.575 & 0.439 & 5.654 \\
\hline AD LAD & 0.254 & 0.875 & 0.084 & 1 & 0.771 & 1.29 & 0.232 & 7.163 \\
\hline LA dilated & 0.661 & 0.541 & 1.494 & 1 & 0.222 & 1.937 & 0.671 & 5.593 \\
\hline MR & -0.253 & 0.664 & 0.145 & 1 & 0.703 & 0.776 & 0.211 & 2.855 \\
\hline Mortality & -0.687 & 0.873 & 0.619 & 1 & 0.431 & 0.503 & 0.091 & 2.785 \\
\hline Constant & -6.705 & 3.276 & 4.19 & 1 & 0.041 & 0.001 & & \\
\hline
\end{tabular}

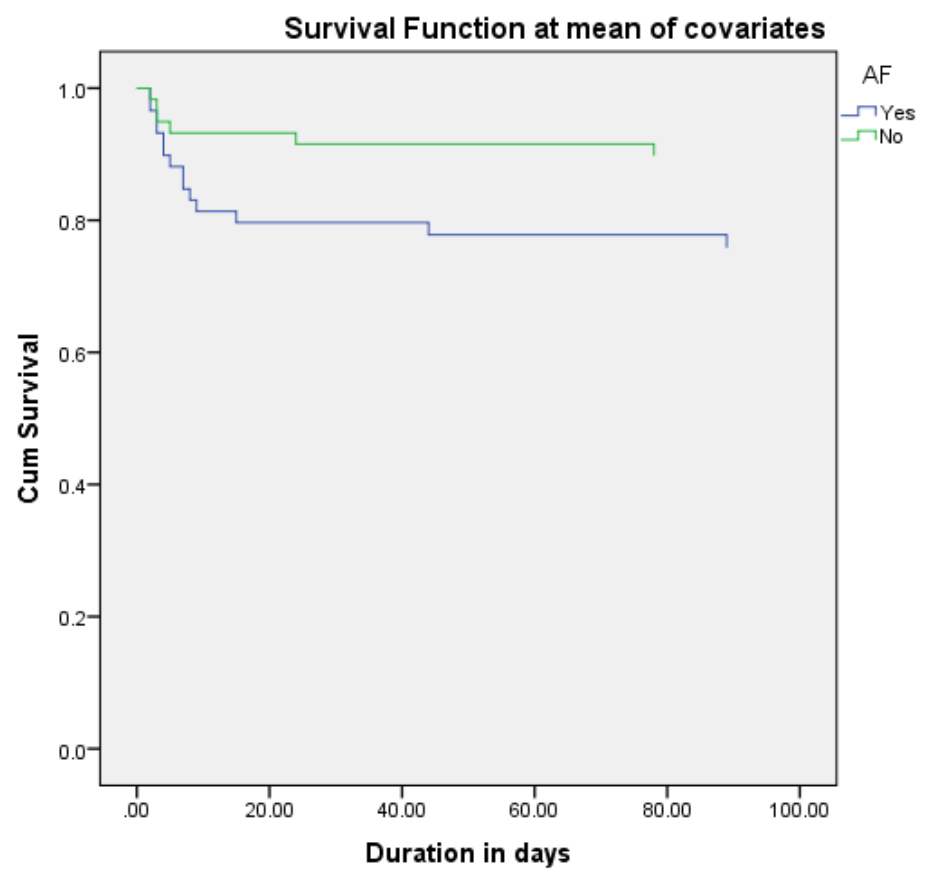




\section{Discussion}

The mechanisms involved in the genesis of atrial fibrillation are mostly focused on ischemia of atrial tissue and hemodynamic change imposed on the atrium due to heart failure ${ }^{12-15}$. Our study noted a higher in-hospital mortality in STEMI patients developing AF.

In the GUSTO I trial ${ }^{5}$ randomly assigning 40891 STEMI patients to thrombolytic therapy with either streptokinase or tPA, patients developing AF had a significantly higher in-hospital mortality. The OPTIMAAL trial ${ }^{10}$ differentiated between patients with AF on admission in whom no statistically significant difference in 30 day mortality was found $(\mathrm{P}=0.27)$, and patients who presented in sinus rhythm and developed $\mathrm{AF}$ during hospitalization. In the latter group of patients, mortality was significantly higher (OR 3.83, 95\% CI 1.97-7.43). Importantly, all patients included in this study had left ventricular dysfunction (measured as LVEF <40\%) in addition to the diagnosis of AMI. In the GISSI -3 trial $^{16}, 1386$ of 17944 (7.8\%) patients with STEMI enrolled during first 24 hours of presentation and receiving optimal treatment with thrombolytics (72\%) and randomized for treatment arms with lisinopril, lisinopril/nitrates and nitrates alone developed AF during their in-hospital stay. In this trial patients with AF had a 1.98 (95\%CI 1.672.34) times increased inpatient mortality risk, which persisted out to 6-months (RR 1.81 95\%CI 1.48-2.23) and 4-year trial period (RR $1.7895 \%$ CI 1.6-1.99).In the primary PCI era trial APEXMI study ${ }^{17}$, which included 5745 AMI patients treated with primary PCI, AF predicted the 90day mortality (HR-1.81, 95\%CI 1.06-3.09) independently of other confounding variables.

Pericarditis complicating STEMI is not uncommon and many such patients develop AF.It is still unclear whether the onset of atrial fibrillation in STEMI with pericarditis is related to pericarditis in itself or to associated haemodynamic change imposed on the atrium by more extensive myocardial damage. In our study we found a higher incidence of AF in patients with pericarditis complicating STEMI.

Acute Kidney Injury (AKI) was more in AF Group than non AF group (16.9\% vs. 5.1\%, $\mathrm{p}=0.04)$. Toshiro Tomomatsu et $\mathrm{al}^{18}$ noticed that the AF group was older had more impaired renal function. Another reason could be the occurrence of more LV dysfunction in AF patients causing renal failure due to cardio-renal syndrome.

Age $>/=70$, systemic hypertension, Left Ventricular $\mathrm{EF}<40 \%$, Left Atrial dilatation, ischemic MR $>/=$ grade 2 were significant predictors of AF on univariate analysis. Hypertension was the only independent predictor of $\mathrm{AF}$ in multivariate analysis. In OACIS study ${ }^{19}, 7.7 \%$ of STEMI patients developed new AF during their inpatient stay and multivariable predictors of arrhythmia development were older age, male gender, HR>100 bpm and Killip class IV.

\section{Conclusions}

More patients having inferior wall STEMI developed AF within 24 hours (59.2\%) . More patients having anterior wall STEMI developed AF after 24 hours (80\%).

A high proportion of STEMI patients who went on to develop acute AF were in Killip's class 3/4 at presentation. One- third of the STEMI patients who developed acute AF also had heart failure.

The in-hospital mortality and MACCE was significantly higher in patients who developed AF. But the 1 and 3 month mortality or MACCE was not significantly different. On comparing anterior wall STEMI with non-anterior wall STEMI, anterior wall STEMI with AF had significantly more in hospital mortality $32.1 \%$ vs. 9.7\% $\mathrm{p}=0.032)$, and in hospital MACCE $(64.3 \%$ vs. $35.5 \%, \mathrm{p}=0.025)$ than non-anterior wall STEMI with AF.

Age $>/=70$, systemic hypertension, Left Ventricular $\mathrm{EF}<40 \%$, Left Atrial dilatation, ischemic mitral regurgitation $>/=$ grade2 were significant predictors of AF on univariate analysis. Hypertension was the only independent predictor of AF in multivariate analysis 
Acute kidney injury was more in the AF group.

\section{References}

1. Eldar M, Canetti M, Rotstein Z, et al. Significance of paroxysmal atrial fibrillation complicating acute myocardial infarction in the thrombolytic era. SPRINT and Thrombolytic Survey Groups. Circulation 1998;97:965-970.

2. 2.Crenshaw BS, Ward SR, Granger CB, Stebbins AL,et al. Atrial fibrillation in the setting of acute myocardial infarction: the GUSTO-I experience. Global Utilization of Streptokinase and TPA for Occluded Coronary Arteries.J Am CollCardiol 1997;30:406-413.

3. Rathore SS, Berger AK, Weinfurt KP, Schulman KA,etal.Acute myocardial infarction complicated by atrial fibrillation in the elderly: prevalence and outcomes. Circulation 2000;101:969-974

4. Wong CK, White HD, Wilcox RG,et al. New atrial fibrillation after acute myocardial infarction independently predicts death: the GUSTO-III experience. Am Heart J 2000;140:878-885.

5. Crenshaw BS, Ward SR, Granger CB et al. Atrial fibrillation in the setting of acute myocardial infarction: the GUSTO-I experience. Global Utilization of Streptokinase and TPA for Occluded Coronary Arteries $J \quad A m$ CollCardiol1997, vol.30 (pg.406 413

6. Kinjo K, Sato H, Sato H, Ohnishi Y, Hishida E, Nakatani D. Prognostic significance of atrial fibrillation/atrial flutter in patients with acute myocardial infarction treated with percutaneous coronary intervention. Am J Cardiol 2003;92:1150-1154.

7. Mehta RH, Dabbous $\mathrm{OH}$, Granger $\mathrm{CB}$, et al. Comparison of outcomes of patients with acute coronary syndromes with and without atrial fibrillation. Am J Cardiol. 2003;92:1031-6.
8. Consuegra-Sanchez L, Melgarejo-Moreno A, Galcera-Tomas J,et al. Short- and longterm prognosis of previous and newonsetatrial fibrillation in ST-segment elevation acute myocardial infarction.Rev EspCardiol. 2015;68:31-8.

9. Podolecki T, Lenarczyk R, Kowalczyk J, et al. Effect of type ofatrial fibrillation on prognosis in acute myocardial infarction treated invasively. Am J Cardiol. 2012;109:1689-93.

10. Lehto M, Snapinn S, Dickstein K, et al. Prognostic risk of atrial fibrillation in acute myocardial infarction complicated by left ventricular dysfunction: the OPTIMAAL experience. Eur Heart J. 2005; 26:350-6.

11. Sakata K, Kurihara H, Iwamori K, et al. Clinical and prognostic significance of atrial fibrillation in acute myocardial infarction. Am J Cardiol.1997; 80:15227.

12. Helmers C, Lundman T, Mogensen L, Orinius E, Sjogren A, Wester PO. Atrial fibrillation in acute myocardial infarction. Acta Med Scand 1973; 193: 39-44.

13. Cristal N, Szwarcberg J, Gueron M. Supraventricular arrhythmias in acute myocardial infarction: Prognostic importance of clinical setting; mechanism of production. Ann Intern Med 1975; 82: $35-9$.

14. Hunt D, Sloman G, Penington C. Effect of atrial fibrillation on prognosis of acute myocardial infarction. Br Heart J 1978; 40: 303-7.

15. Sugiura T, Iwasaka T, Ogawa A et al. Atrial fibrillation imyocardial infarction. Am JCardiol.1997; 80:15227.

16. Pizzetti F, Turazza FM, Franzosi MG, et al. GISSI-3 Investigators. Incidence and prognostic significance of atrial fibrillation in acute myocardial infarction: the GISSI3 data. Heart. 2001;86:527-32.

17. Lopes RD, Elliott LE, White HD, et al. Antithrombotic therapy and outcomes of 
patients with atrial fibrillation following primary percutaneous coronary intervention: results from the APEX-AMI trial. Eur Heart J. 2009;30:2019-28.

18. Toshiro Tomomatsu, ItsuroMorishima, TakahitoSone, New Onset At rial Fibrillation, But Not Pre-existing The Arrhythmia, May Be An Independent Predictor Of Cardiac Mortality In Patients With Acute Myocardial Infarction; JACC March 12, 2013 Volume 61, Issue 10

19. Kinjo K, Sato H, Sato H, et al. Osaka Acute Coronary Insufficiency Study (OACIS) Group. Prognostic significance of atrial fibrillation/atrial flutter in patients with acute myocardial infarction treated with percutaneous coronary intervention. Am J Cardiol. 2003;92:1150-4. 